\title{
Optically Polarized Conduction-Band Electrons in Tungsten Observed by Spin- Polarized Photoemission
}

\author{
Zürcher, P.; Meier, F.; Christensen, N. E.
}

Published in:

Physical Review Letters

Link to article, DOI:

10.1103/PhysRevLett.43.54

Publication date:

1979

Document Version

Publisher's PDF, also known as Version of record

Link back to DTU Orbit

Citation (APA):

Zürcher, P., Meier, F., \& Christensen, N. E. (1979). Optically Polarized Conduction-Band Electrons in Tungsten Observed by Spin-Polarized Photoemission. Physical Review Letters, 43(1), 54-57.

https://doi.org/10.1103/PhysRevLett.43.54

\section{General rights}

Copyright and moral rights for the publications made accessible in the public portal are retained by the authors and/or other copyright owners and it is a condition of accessing publications that users recognise and abide by the legal requirements associated with these rights.

- Users may download and print one copy of any publication from the public portal for the purpose of private study or research.

- You may not further distribute the material or use it for any profit-making activity or commercial gain

- You may freely distribute the URL identifying the publication in the public portal

If you believe that this document breaches copyright please contact us providing details, and we will remove access to the work immediately and investigate your claim. 


\footnotetext{
${ }^{2}$ F. C. Frank, Discuss. Faraday Soc. $\underline{25}, 19$ (1958).

${ }^{3}$ Ping Sheng, Phys. Rev. Lett. 37, 1059 (1976);

H. Schröder, J. Chem. Phys. 67, 16 (1977); R. B. Meyer, in Molecular Fluids, edited by R. Balian and G. Weill (Gordon Breach, London, 1976).

${ }^{4}$ P. G. de Gennes, The Physics of Liquid Crystals (Oxford, London, 1974).

${ }^{5}$ From Ref. 3 this is a reasonable assumption for a film thicker than $1 \mu \mathrm{m}$.

${ }^{6}$ H. J. Coles, Mol. Cryst. Liq. Cryst. $\underline{49}, 67$ (1978).
}

\author{
${ }^{7}$ T. W. Stinson and J. D. Litster, Phys. Rev. Lett. $\underline{30}$, \\ 688 (1973). \\ ${ }^{8}$ I. Haller, J. Chem. Phys. 57, 1400 (1972). \\ ${ }^{9}$ M. Born and E. Wolf, Principles of Optics, (Perga- \\ mon, London, 1970), p。699. \\ ${ }^{10}$ R. G. Horn, J. Phys. (Paris) $\underline{39}, 105$ (1978). \\ ${ }^{11}$ J. L. Janning, Appl. Phys. Lett. 21,173 (1972). \\ ${ }^{12}$ W. Urbach, M. Boix, and E. Guyon, Appl. Phys. \\ Lett. 25,479 (1974)。 \\ ${ }^{13}$ D.W.Berreman, Phys。Rev。Lett. $\underline{28}, 1683$ (1972).
}

\title{
Optically Polarized Conduction-Band Electrons in Tungsten Observed by Spin-Polarized Photoemission
}

\author{
P. Zürcher and F. Meier \\ Laboratorium für Festkörperphysik, Eidgeno̊ssische Technische Hochschule Zürich, \\ $\mathrm{CH}-8093$ Zürich, Switzerland \\ and \\ N. E. Christensen \\ Physics Laboratory I, The Technical University of Denmark, Copenhagen DK-2800, Denmark
}

(Received 19 March 1979)

\begin{abstract}
Along the (100) direction of tungsten, interband transitions induced by circularly polarized light of energy $1.5 \mathrm{eV} \leqslant h \nu \leqslant 4.1 \mathrm{eV}$ lead to spin polarization of the excited conduction electrons. This is observed by measuring the spin polarization of the photoelectrons emitted from a cesiated crystal surface. The measured polarization values depend sensitively on the $s-, p^{-}$, and $d$-hybridization properties of the conduction bands. Excitedstate lifetimes are obtained from the widths of the polarization structures.
\end{abstract}

The now widespread use of synchrotron radiation in angular resolved photoemission has made it increasingly clear that the optical selection rules for linearly polarized light provide an elegant means for identifying the parity of electronic states. ${ }^{1}$ In this paper we show that the symmetry properties of the electronic wave functions can be investigated in further detail by photoemission using circularly polarized light. The present technique requires the measurement of the spin polarization of the photoelectrons.

The experiment is based on the fact that unpolarized electrons can be excited by circularly polarized light into spin-polarized final states. Although such optical polarization experiments have been made earlier with use of selected transitions in semiconductors, ${ }^{2,3}$ only the present measurements with tungsten indicate that final-state polarization can be obtained under very general conditions also for metals. ${ }^{4}$ Therefore it appears worthwhile to explore the possibilities of this method systematically. As it relies on the selection rules for circularly polarized light, the symmetry of the electronic states is of crucial importance. A decomposition into spin orbitals of given $l$ value ( $l$ is the orbital angular momentum quantum number) is useful. The symmetry properties of the spin orbitals are found from group theory. The spin polarization in each excited state is determined by the mixing of the spin"up" and -"down" components in this state.

Essential points of the experiment are as follows: (i) The axial vector defining the direction of the final-state spin polarization is given by the spin direction of the circularly polarized light. (ii) In the absence of spin-orbit coupling the electronic wave functions are pure spin states. Then, because light interacts only with the orbital motion of the electrons, spin-up and -down electrons participate identically in all transitions and no overall spin polarization is obtained. Therefore spin mixing by spin-orbit interaction in at least one of the states involved in a given transition is necessary for final-state spin polarization. (iii) Simple symmetry considerations show that for crystals with inversion symmetry, as, e.g., tungsten, transitions produced by rightand left-circularly polarized light have the same absolute values of the polarization but opposite signs. 
Tungsten was chosen for this experiment because it shows appreciable spin-orbit interaction, ${ }^{5}$ is readily available as single crystals, ${ }^{6}$ is easy to clean, and has a band structure that is quite well known. ${ }^{5}$ Our specimen was a W(100) single-crystal platelet of $5 \mathrm{~mm}$ diam. It was cleaned by up to twenty successive cycles consisting of heat treatment at $1800^{\circ} \mathrm{C}$ (duration 30 sec) followed by exposure to $5 \times 10^{-6}$ Torr of oxy gen during $2.5 \mathrm{~min}$ at $1600^{\circ} \mathrm{C}$. As final step, after the last oxygen exposure, the carbon monoxide and sulfur oxide were evaporated by flash heating to $2100^{\circ} \mathrm{C}$. The surface condition was checked by low-energy-electron-diffraction (LEED)-Auger analysis. The LEED pattern was identical to the one reported by Melmed, Layer, and Kruger ${ }^{7}$ for a clean W(100) surface. Traces of sulfur could still be detected in the Auger signal, whereas the carbon signal was very weak and no indication of oxygen could be found. In order to investigate the band structure at low energies, $h \nu<5 \mathrm{eV}$, the crystals were cesiated to reduce the work function. A detailed account of the experimental setup to measure the spin polarization has been given before. ${ }^{8}$ The measurements were done at a pressure of $4 \times 10^{-10}$ Torr with the sample held at a temperature of $-40^{\circ} \mathrm{C}$.

The polarization spectra for two samples with work function $\Phi=1.5 \mathrm{eV}$ and $\Phi=2.4 \mathrm{eV}$ are shown in Fig. 1. At photon energies $h \nu=2.1 \mathrm{eV}$ and $h \nu$ $=2.9 \mathrm{eV}$ maxima of the polarization, $-6 \%$ and $+7.5 \%$, respectively, are observed for the lowwork-function sample. These transitions are denoted by $T_{1}$ and $T_{2}$. A comparison of the two spectra shows that both are identical in the common range of photon energies.

A theoretical prediction of the polarization spectrum has been published by Reyes and Helman. ${ }^{9}$ From group theory the symmetry of the $s-p-$ and $d$-derived conduction-band states is determined along the normal to the $\mathrm{W}(100)$ face, i.e., the $\Delta$ direction. Then the allowed optical transitions are found by applying the selection rules for circularly polarized light. The predicted high polarizations $\left(-100 \%\right.$ for $T_{1},+100 \%$ for $T_{2}$ ) could not be observed. There is a sign reversal of the polarization at $h \nu=2.4 \mathrm{eV}$ in qualitative agreement with Ref. 9 but it occurs at a photon energy $0.4 \mathrm{eV}$ higher than predicted. The crossing point of the polarization spectra with the $P=0$ line was reproducible within $60 \mathrm{meV}$ in six experiments.

The discussion of these discrepancies must take into account that the theory was developed exclusively for transitions along $\Delta$, whereas in

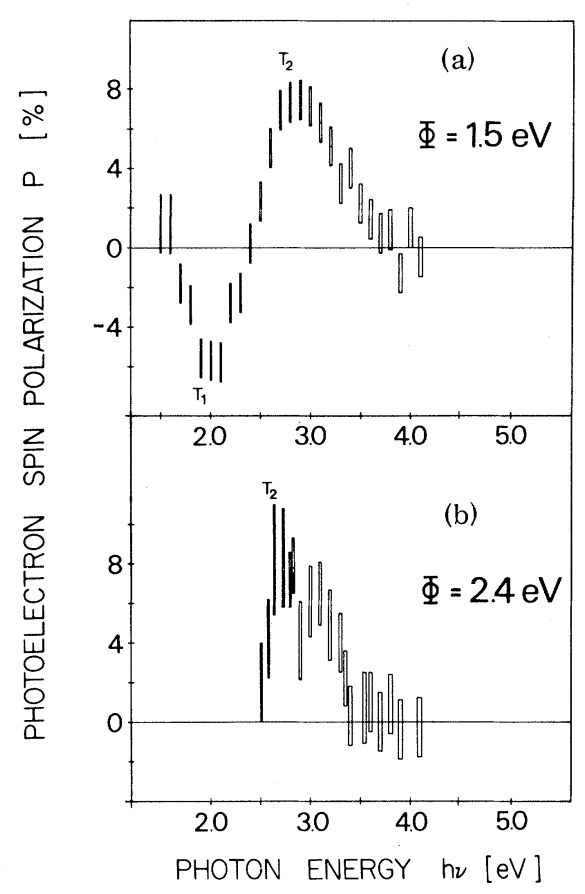

FIG. 1. W(100) single-crystal surface: Dependence of the photoelectron spin polarization $P$ on photon energy for two cesiated samples with work functions (a) $\Phi=1.5 \mathrm{eV}$ and (b) $\Phi=2.4 \mathrm{eV}$.

the experiment the acceptance angle was not externally restricted. We observe, however, that in the present case the escape cone does not influence the absolute value of the spin polarization. The spectra of the two samples with different work functions and hence with different escape cones (Fig. 1) look, indeed, very similar in the common $h \nu$ range. This is consistent with the calculated band structure of tungsten ${ }^{5}$ which shows that the escape cone for the $T_{1}$ and $T_{2}$ transitions is less than $\pm 14^{\circ}$.

From Fig. 1 we deduce that $T_{1}$ and $T_{2}$ are due to direct transitions since $T_{1}$ is simply cut off when the work function is increased from 1.5 to $2.4 \mathrm{eV}$. This is a further proof that direct bulk transitions occur in tungsten. ${ }^{10}$ It also makes clear that the $T_{2}$ structure is not due to transitions near $\Gamma\left(\Delta_{6,7} \rightarrow \Delta_{7}\right)$ because $T_{2}$ should then also vanish upon increasing the vacuum level to $2.4 \mathrm{eV}$

The only effect which we attribute to the escape cone is that the measured maxima of the polarization occur at energies $0.3-0.5 \mathrm{eV}$ higher than expected from Fig. 2. The shape of the energy surfaces near the $\Delta$ direction and the corresponding partial joint density of states (PJDOS) (see 
Fig. 3) cause the maximum transition probability to occur at light energies slightly higher than those needed for transitions on the $\Delta$ line. This also explains the position of the zero crossing in Fig. 1(a) to within $\sim 0.2 \mathrm{eV}$, which is probably about the accuracy of the band-structure calculation.

Although the transitions responsible for the polarization structure can be unambiguously identified in Fig. 2, the interpretation of the degree of polarization produced by these transitions is a more difficult problem. Depolarization of the photoelectrons by spin-exchange scattering with the cesium on the surface is negligible. The sample with $\Phi=2.4 \mathrm{eV}$ is less cesiated than the one with $\Phi=1.5 \mathrm{eV}$. Nevertheless the polarization of the $T_{2}$ transition is the same in both cases. This is not surprising because only 0.25 of a cesium monolayer is necessary to obtain $\Phi=1.5 \mathrm{eV} .^{11}$ At these low coverages the cesium is ionized and has a rare-gas electron configuration with zero spin. Also measurements with slightly cesiated nickel and $\mathrm{GaAs}$ never gave a clear indication for depolarization. ${ }^{2,12}$

In addition to the bulk interband transitions $T_{1}$

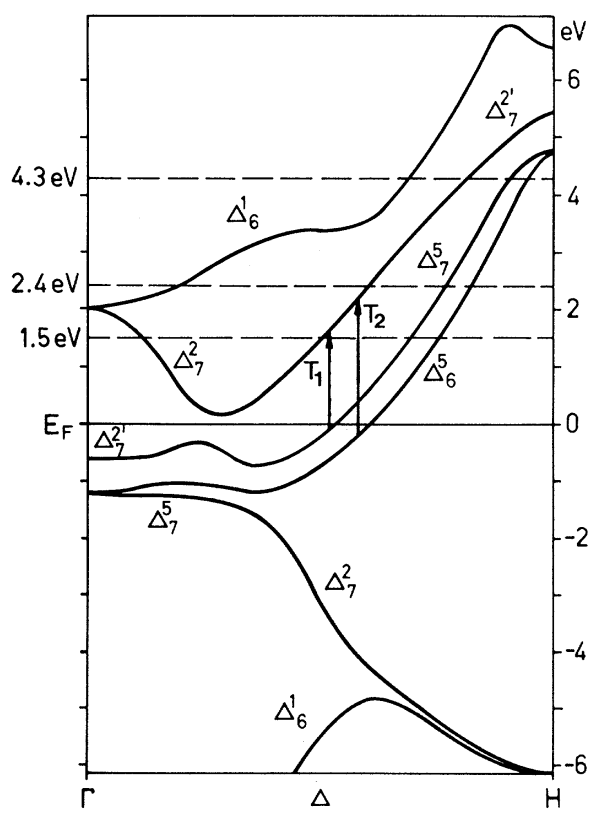

FIG. 2. Electronic energy-band structure of tungsten for the (100) direction according to Ref. 5. The vacuum levels for the free surface $(4.3 \mathrm{eV})$ and the two cesiated surfaces $(2.4$ and $1.5 \mathrm{eV})$ are indicated. Because of the curvature of the energy bands near $\Gamma-H$, the finite escape cone, and the lifetime broadening, the $T_{2}$ transition is also observed for the $2.4-\mathrm{eV}$ phothothreshold. and $T_{2}$ there are photoelectrons produced by direct emission usually referred to as surface photoemission. In the present experiment this surface emission is unpolarized as can be seen from Fig. 1. For instance, the $\varphi=1.5 \mathrm{eV}$ sample shows surface emission near threshold because no emitting interband transitions are possible at this energy; see Fig. 3.

Also at light energies above threshold the spectra of Fig. 1 give no indication of polarized surface emission. As a continuum of final states is available in surface emission any polarization structure would depend on $h \nu-\varphi$ alone and not on the details of the band structure. However, the spectra shown in Fig. 1 are unambiguously due to bulk interband transitions. In particular, the polarization peak $T_{2}$ occurs at a well-defined light energy independent of the value of the photothreshold.

Also at higher photon energies surface emission makes a large contribution to the total photocurrent. For the three high-symmetry surfaces of tungsten the interband transitions at $h \nu=10.2$ $\mathrm{eV}$ are superposed on a background of surface emission which amounts to $70-85 \%$ of the total photocurrent. ${ }^{10}$ The surface emission is proportional to the surface density of states which is well known for tungsten. ${ }^{10}$ The PJDOS giving rise to $T_{1}$ and $T_{2}$ shown in Fig. 3 takes into account the acceptance angle of the apparatus and the requirements that the initial state be below the Fer$\mathrm{mi}$ level and the final state above the vacuum level. Figure 3 shows that the PJDOS of $T_{1}$ and $T_{2}$ is quite insensitive as to whether the acceptance

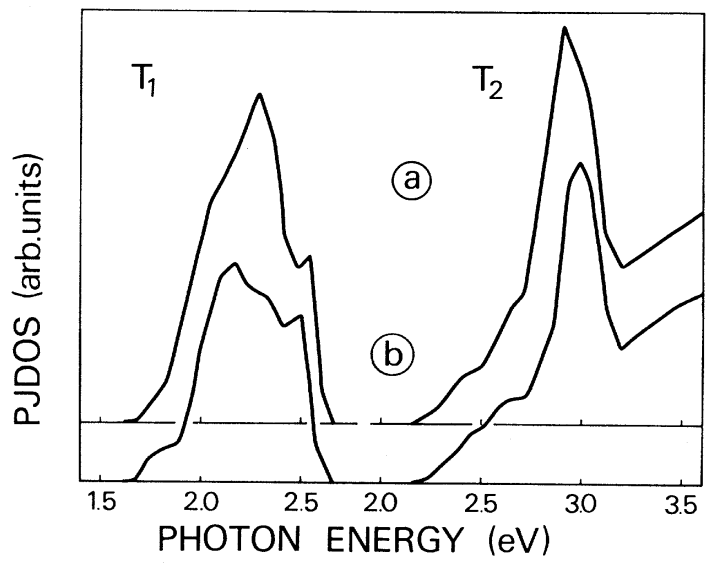

FIG. 3. Partial joint density-of-states (PJDOS) functions of $\mathrm{W}(100)$ for the transitions $T_{1}$ and $T_{2}$ in a sample with work function $1.5 \mathrm{eV}$, for an acceptance angle of (a) $\pm 90^{\circ}$, (b) $\pm 45^{\circ}$. 
angle is $\pm 45^{\circ}$ or $\pm 90^{\circ}$. Therefore the ratio of surface to total (= surface + bulk) emission can be estimated for cesiated tungsten at photon energies corresponding to $T_{1}$ and $T_{2}$. Average values of this ratio are taken over the widths of the transitions $T_{1}$ and $T_{2}$. For $1.7 \mathrm{eV} \leqslant h \nu \leqslant 2.2$ and 2.3 $\mathrm{eV} \leqslant h \nu \leqslant 3.2 \mathrm{eV}$ they are found to be $(49 \pm 5) \%$ and $(75 \pm 4) \%$, respectively. Allowing for this unpolarized surface emission and the small overlap of $T_{1}$ and $T_{2}$ due to lifetime broadening, the initial polarizations $P_{1}$ and $P_{2}$ of $T_{1}$ and $T_{2}$ are estimated to be $(-16 \pm 6) \%$ and $(32 \pm 10) \%$, respectively. This is still far off the theoretically predicted $\pm 100 \%$. In Ref. 9 the final-state $\Delta_{7}$ band of the $T_{1}$ and $T_{2}$ transitions is assumed to be purely $d$ type. This is questionable since strong hybridization with $p$-type functions of proper symmetry is expected near the band-crossing region. We then conclude that it is this hybridization which reduces the polarization drastically. The $\overrightarrow{\mathrm{k}}-\mathrm{de}-$ pendent hybridization of the $\Delta_{7}{ }^{5}$ and $\Delta_{7}{ }^{2}$ wave functions in the final state of $T_{1}$ and $T_{2}$ and in the initial state of $T_{1}$ is estimated from the relation

$$
a=2 P^{-1}\left[P+1-\left(1-P^{2}\right)^{1 / 2}\right],
$$

where $a$ and $(1-a)$ are the $\Delta_{7}{ }^{\prime}$ and $\Delta_{7}{ }^{5}$ contributions to the final-state wave functions, respectively: With the initial polarizations $P_{1}$ and $P_{2}$ one obtains the following results: For $\overrightarrow{\mathrm{k}}$ values corresponding to the $T_{1}$ transition the final state is $(54 \pm 2) \% d$ type of $\Delta_{7}{ }^{\prime}{ }^{\prime}$ symmetry and $(46 \pm 2) \%$ $p$ and $d$ type of $\Delta_{7}{ }^{5}$ symmetry. At larger $\overrightarrow{\mathrm{k}}$ values belonging to the $T_{2}$ transition the corresponding values are $(58.5 \pm 2.5) \%$ and $(41.5 \pm 2.5) \%$, respectively.

For the $\Delta_{7}{ }^{5}$ initial states the $d$ and $p d$ contributions are interchanged, whereas for the $\Delta_{6}{ }^{5}$ initial states it is not possible to obtain the hybridization from the electron-spin-polarization results presented in this Letter. With regard to these hybridized states, the change of sign of the polarization of the $T_{1}$ and $T_{2}$ transitions is due to the double degeneration of the states by time-reversal symmetry. Whereas one of these states is excited by $T_{1}$, it is the time-reversed state with the other sign of polarization which is excited by $T_{2}$.

The widths of the measured polarization peaks $T_{1}$ and $T_{2}$ can be used to determine the lifetime of the excited states. This method supplements one based on energy distribution curves ${ }^{13}$ but requires the measurement of only one spectrum. In order to avoid effects due to overlapping tran- sitions only the low-energy side of the peaks is used to determine the full width at half maximum, $\Gamma$. For $T_{1}$ we obtain $\Gamma=0.35 \pm 0.09 \mathrm{eV}$ and for $T_{2}$ the value is $\Gamma=0.52 \pm 0.10 \mathrm{eV}$. The error mar gins are derived from the estimated uncertainty of the calculated surface emission, the uncertainty of the widths of the measured polarization peaks, and the statistical errors of the measured polarization values. The corresponding lifetimes are $(2.0 \pm 0.5) \times 10^{-15} \mathrm{sec}$ and $(1.3 \pm 0.25)$ $\times 10^{-15} \mathrm{sec}$. From knowledge of the group velocity of the excited electrons ${ }^{5}$ the escape depths $l(E)$, where $E$ is the final-state energy above the Fermi level, are obtained. For the $T_{1}$ transition $(E=1.95 \mathrm{eV})$ it is $14 \pm 4 \AA$ and for the $T_{2}$ transition $(E=2.35 \mathrm{eV}), 9 \pm 2 \AA$. These values fill a sparsely documented part of the well-known universal $l(E)$ curve. ${ }^{14}$

We thank H. C. Siegmann for his continuous interest in this work, A. J. Freeman for many fruitful suggestions, and P. S. Bagus, W. Eib, and M. Landolt for stimulating discussions. We are grateful to $\mathrm{B}$. Feuerbacher for a detailed description of the W-cleaning procedure. Financial support by the Schweizerische Nationalfonds is gratefully acknowledged.

\footnotetext{
${ }^{1}$ E. Dietz and D. E. Eastman, Phys. Rev. Lett. $\underline{41}$, 1674 (1978).

${ }^{2}$ D. T. Pierce and F. Meier, Phys. Rev. B $\underline{13}, 5484$ (1976).

${ }^{3} \mathrm{P}$. Zürcher and F. Meier, to be published.

${ }^{4}$ Emission of polarized electrons from alkali metal films, although not under uhv conditions, was observed by U. Heinzmann et al., Z. Phys. 251, 354 (1972).

${ }^{5}$ N. E. Christensen and B. Feuerbacher, Phys. Rev. B 10, 2349 (1974).

${ }^{6}$ Materials Research GmbH, 8000 München 70, Pollingerstrasse, 5, Germany.

${ }^{7}$ A. J. Melmed, H. P. Layer, and J. Kruger, Surf. Sci. 9,476 (1968).

${ }^{8}$ S. F. Alvardo, W. Eib, F. Meier, H. C. Siegmann, and P. Zürcher, in Photoemission and the Electronic Properties of Surfaces, edited by B. Feuerbacher, B. Fitton, and R. F. Willis (Wiley, New York, 1978), p. 437 .

${ }^{9}$ J. Reyes and J. S. Helman, Phys. Rev. B $\underline{16}, 4283$ (1977).

${ }^{10} \mathrm{~B}$. Feuerbacher and N. E. Christensen, Phys. Rev. B 10, 2373 (1974).

${ }^{11} \mathrm{C}$. S. Wang, J. Appl. Phys. 48, 1477 (1977).

${ }^{12}$ W. Eib, Eidgenössische Technische Hochschule disseration No.5978, 1978 (unpublished)。

${ }^{13}$ D. E. Eastman, J. A. Knapp, and F. J. Himpsel, Phys. Rev. Lett. $\underline{41}, 825$ (1978).

${ }^{14}$ I. Lindau and W. E. Spicer, J. Electron Spectrosc. Relat. Phenom. $\underline{3}, 409$ (1974).
} 